\title{
Bismuth Hexagons: Facile Mass Synthesis, Stability and Applications
}

\author{
Tirtha Som,${ }^{[\mathrm{a}]}$ Anne Simo, ${ }^{[\mathrm{a}]}$ Robert Fenger, ${ }^{[\mathrm{a}]}$ Gerald V. Troppenz, ${ }^{[\mathrm{b}]}$ Roman \\ Bansen, ${ }^{[\mathrm{c}]}$ Norbert Pfänder, ${ }^{[\mathrm{d}]}$ Franziska Emmerling,${ }^{[\mathrm{e}]}$ Jörg Rappich, ${ }^{[\mathrm{b}]}$ Torsten \\ Boeck, ${ }^{[\mathrm{c}]}$ and Klaus Rademann*[a]
}

((Dedication, optional))

A unique direct electrodeposition technique involving very high current densities, high voltages and high electrolyte concentrations has been applied for highly selective mass synthesis of stable, isolable, surfactant-free, single-crystalline $\mathrm{Bi}$ hexagons on a $\mathrm{Cu}$ wire at room temperature. A formation mechanism of hexagons has been proposed. The morphology, phase purity, and crystallinity of the material have been well characterized by FESEM, AFM, TEM, SAED, $E D X, X R D$, and Raman spectroscopy. The thermal stability of the material under intense electron beam and intense laser light irradiation has been studied. The chemical stability of elemental Bi in

\section{Introduction}

Synthesis of high-quality crystalline semi-metals in reduced dimensions has emerged as one of the most active areas of current materials research. ${ }^{[1]}$ The dimensionally restricted semimetals and their different morphologies lead to exciting physical and chemical phenomena, very different from those of the typical noble and transition metals. These semi-metals with unique electronic properties exhibit adjustable band gaps, and distinctive surface and bulk transport properties (topological insulators). They also have the unique ability to directly convert heat to electricity (thermoelectric materials or alternative photovoltaic materials). ${ }^{[2]}$ Consequently, they are highly desirable materials for advanced next-generation optical, electronic, thermoelectrical, catalytic and mechanical applications. The diamagnetic semimetal $\mathrm{Bi}$ and its inter-metallic alloys hold great promise in all these applications and have evolved into a special family of semiconducting materials. ${ }^{[1-3]}$

$\mathrm{Bi}$ has been the first thermoelectric material to be studied and found applications in radiation thermopiles. ${ }^{[4]}$ Indeed the first three-dimensional topological insulator is also based on Sbdoped $\mathrm{Bi}$ where the surface is metallic and the bulk is insulating or semiconducting depending on the thickness. ${ }^{[3 a]} \mathrm{Bi}$ crystallizes in rhombohedral structure with two atoms per unit cell. ${ }^{[5]}$ A small distortion of atomic positions accompanied by spin-orbit interactions induces a lowering of the conduction band and thereby an indirect negative band gap. Also, at some points of the Brillouin zone there are small overlaps $(\sim 40 \mathrm{meV})$ between the conduction and valence bands. ${ }^{[5]}$ So $\mathrm{Bi}$ with effective carrier nitric acid shows different dissolution rates for different morphologies. This effect enables a second way for the selective fabrication of $\mathrm{Bi}$ hexagons. $\mathrm{Bi}$ hexagons can be oxidized exclusively to $\alpha-\mathrm{Bi}_{2} \mathrm{O}_{3}$ hexagons. The $B i$ hexagons are found to be promising for thermoelectric applications. They are also catalytically active inducing the reduction of 4-nitrophenol to 4-aminophenol. This electrodeposition methodology has also been demonstrated to be applicable for synthesis of bismuth-based bimetallic hybrid composites for advanced applications.

mobility, at reduced dimensions offers the possibility to study the metal-to-semiconductor transition and quantum-confined effects, like quantum-confined Stark effect. ${ }^{[6]}$ It leads to the evolution of a plethora of new and exciting physical phenomena like very high magnetoresistance, high thermoelectric efficiency, and low temperature superconductivity. ${ }^{[1 \mathrm{a}, 7]} \mathrm{Bi}$ also induces chemical phenomena like catalysing the growth of semiconductor nanowires like $\mathrm{SnS}_{2}, \mathrm{Ge}$, or $\mathrm{CdSe}^{[8]}$ It induces oxidation processes of unsaturated aliphatic compounds ${ }^{[9]}$ and reversible photochromic effects. ${ }^{[10]}$ It is also used for detection of trace elements (like $\mathrm{Cd}^{2+}, \mathrm{Pb}^{2+}, \mathrm{Ni}^{2+}, \mathrm{Al}^{3+}$ ) in an aqueous medium. ${ }^{[11,12 a]}$

The morphology and microstructure of a material plays a crucial role in determining its physical properties. A variety of distinct morphologies of Bi like micro and nanospheres, nanorods, nanotubes, nanowires, nanocubes, nano-branches, nanotriangles,

[a] Dr. T. Som, A. Simo, R. Fenger, Prof. Dr. K. Rademann Institut für Chemie

Humboldt-Universität zu Berlin

Brook-Taylor-Strasse 2, 12489 Berlin (Germany)

Fax: (+49) 30-2093-555

E-mail: klaus.rademann@chemie.hu-berlin.de

[b] G. V. Troppenz, Dr. J. Rappich Institut für Silizium-Photovoltaik Helmholtz-Zentrum Berlin Kekuléstrasse 5, 12489 Berlin (Germany)

[c] R. Bansen, Dr. T. Boeck Leibniz-Institut für Kristallzüchtung Max-Born-Strasse 2, 12489 Berlin (Germany)

[d] N. Pfänder

Fritz-Haber-Institut der Max-Plank-Gesellschaft Faradayweg 4-6, 14195 Berlin (Germany)

[e] Dr. F. Emmerling

BAM Federal Institute of Materials Research and Testing Richard-Willstätter-Strasse 11, 12489 Berlin (Germany) 
island films, star-shapes, dendrites have been generated by various techniques like chemical, solvothermal, hydrothermal synthesis, electron-beam irradiation, electron beam lithography, vapour deposition and electrodeposition. ${ }^{[56,12-15]}$ Particular emphasis has been given to synthesis of single-crystalline materials which are expected to exhibit several enhanced physical phenomena. ${ }^{[15]}$ Yarema et al ${ }^{[13 a]}$ have demonstrated the self-assembly of highly uniform spherical $\mathrm{Bi}$ surfactant-aided colloidal nanoparticles into long-range-ordered two and threedimensional superstructures like hexagonal-prism, octahedral and truncated octahedron over a long duration. Wang et al[ ${ }^{13 b]}$ also showed the evidence of existence hexagonal morphology of $\mathrm{Bi}$ in a mixture of nanorods and dots in solution phase polymer assisted synthesis. However, a quick, direct, single-step fabrication of pure $\mathrm{Bi}$ hexagonal platelets under surfactant-free conditions has never been reported before, although hexagonal particles of $\mathrm{Bi}_{2} \mathrm{Te}_{3}, \mathrm{BiNi}$ and $\mathrm{BiOCl}$ are widely known. ${ }^{[16]}$

In this article, being motivated by all of the mentioned $\mathrm{Bi}$ based features, we report the highly selective mass synthesis of stable, isolable, surfactant-free, single-crystalline $\mathrm{Bi}$ hexagons and explore the potential applications of this morphology in thermoelectrics and catalysis.

\section{Results and Discussion}

\section{Fabrication}

The Bi hexagons have been obtained via a facile electrochemical deposition route at room temperature and ambient atmosphere, employing high current densities $\left(180 \mathrm{mAmm}^{-2}\right)$, strong cathodic overpotentials (typically $-10 \mathrm{~V}$ ) far away from the standard reduction potential of $\mathrm{Bi}^{3+}: \mathrm{Bi}^{3+}+3 \mathrm{e}^{-} \rightarrow \mathrm{Bi}^{0}, \mathrm{E}^{0}=0.308 \mathrm{~V} \cdot{ }^{[17]}$ Most importantly we employed high concentrations of the reactant $\mathrm{Bi}\left(\mathrm{NO}_{3}\right)_{3} \cdot 5 \mathrm{H}_{2} \mathrm{O}(0.5 \mathrm{M})$ dissolved in $1 \mathrm{M} \mathrm{HNO}_{3}$ solution, which served as the electrolyte. Over the past decade, electrodeposition has evolved into a more powerful and successful room temperature facile strategy to fabricate mesoscopic or even nanoscopic metallic structures. ${ }^{[12,18]}$ In fact, electrodeposition has been widely used to decorate various template surfaces by a variety of elements including $\mathrm{Bi}^{\left[{ }^{[12,18]}\right.}$ However, the formation of $\mathrm{Bi}$ hexagons via the electrodeposition method has never been reported before in literature. This might be because electrodeposition is usually carried out at near equilibrium potentials, but rarely at exceedingly strong cathodic overpotentials. Our new synthesis procedure, employing high potentials and high concentrations, ensures the mass fabrication of $\mathrm{Bi}$ hexagons within very short deposition times in very strong electrolytes (acids). The short deposition times effectively suppress any competing redissolution processes, which definitely occur in nitric acid on longer time scales.

The working electrode has been a $\mathrm{Cu}$ wire of diameter 0.4 $\mathrm{mm}$ and length of about $2.5 \mathrm{~mm}$ as the cathode. A Bi metallic rod $(10 \mathrm{~mm})$ is used as the anode. With a galvanostatic deposition time of $30 \mathrm{~s}$, some flower-like dendritic structures deposit on the $\mathrm{Cu}$ wire (Figure 1a). However, careful examination of these structures with a high resolution field emission scanning electron microscope (FESEM) reveals that these dendrites actually comprise of hexagons. Distinct small hexagons of major axis in the range of $0.4-1 \mu \mathrm{m}$ can be identified at the tip of the dendrites (Figure 1b).

With further increase in deposition time to $1 \mathrm{~min}$, large sized non-porous hexagonal structures of about $10-20 \mu \mathrm{m}$ grow out from the stems of the dendrites, as becomes evident from the environmental scanning electron microscopic (E-SEM) images (Figure 1c and d). The obtained microstructures are highly dense, three-dimensional, symmetrical, almost parallel and uniformly arranged relative to the main trunk (Figure 1c and d). The thickness is estimated to be about $1 \mu \mathrm{m}$ or less. When the deposition time is $2.5 \mathrm{~min}$, large hexagons having sizes in the range of $95-150 \mu \mathrm{m}$ and thickness of about $3 \mu \mathrm{m}$ grow in an upright fashion on the wire (Figure 2). The hexagonal morphology is retained but the size and thickness of the hexagons increases with deposition time due to enhanced electrodeposition of $\mathrm{Bi}^{3+}$ ions on elemental $\mathrm{Bi}$. These larger $\mathrm{Bi}$ hexagons are black shiny electrodeposits. So they may be used as components of special effect glossy pigments in addition to $\mathrm{BiOCl}^{\left[{ }^{[16 \mathrm{~d}]}\right.}$ They can be easily washed thoroughly by dipping them into de-ionized water. They can be isolated from the $\mathrm{Cu}$ wire by scratching with a blade and can be used for further characterization and application studies. It is found that $\mathrm{Bi}$ structures continue to grow with time on the predeposited $\mathrm{Bi}$ in a tree-like fashion, which indicates the electrical conductivity and continuity of the electro-generated $\mathrm{Bi}$ hexagons.
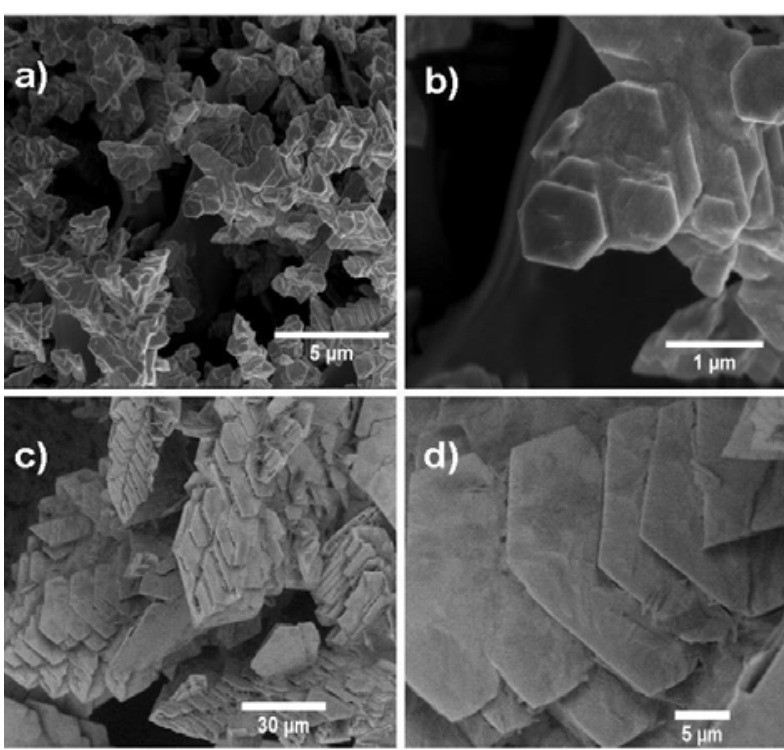

Figure 1. a) and b) FESEM images of Bi structures electrodeposited for 30s, c) and d) E-SEM images of Bi structures electrodeposited for 1 min

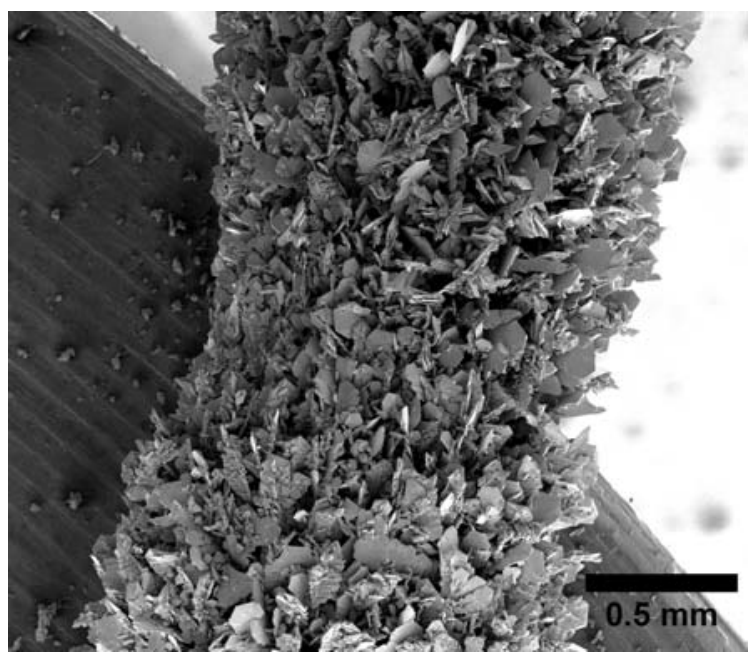

Figure 2. FESEM image of isolable upright large $\mathrm{Bi}$ hexagons growing on the $\mathrm{Cu}$ wire when galvanostatic deposition time is $2.5 \mathrm{~min}$ 
We also found that galvanostatic electrodeposition of $\mathrm{Bi}$ can be carried out on a variety of surfaces like graphite, $\mathrm{Al}, \mathrm{Au}, \mathrm{Ag}$ and $\mathrm{Si}$. Lower current densities $\left(10-75 \mathrm{mAmm}^{-2}\right)$, lower voltages $(-2 \mathrm{~V}$ to $-8 \mathrm{~V})$, shorter deposition times $(5-10 \mathrm{~s})$ and lower concentrations $\left(10^{-3}-10^{-1} \mathrm{M}\right)$ results in non-uniform morphologies and many irregular shaped polygons (Figure 3). Under these conditions, there are also the developments of sparsely spaced fern-shaped $\mathrm{Bi}$ dendrites, similar to the observation by Yang, ${ }^{[12 b]}$ and barbed wire fences with sharp edges oriented at right angles arranged at regular intervals along the strand. Only a few isolated small polycrystalline hexagons are generated under these conditions.

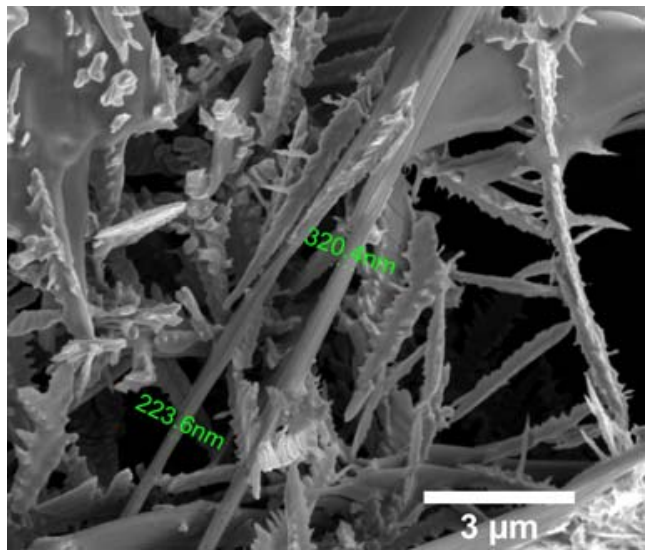

Figure 3. FESEM image showing Bi polygons and partly barbed and nonbarbed nano wires with typical diameters between 220 - $320 \mathrm{~nm}$. These preliminary structures are formed by slow reduction of $\mathrm{Bi}^{3+}$ ions to $\mathrm{Bi}^{0}$, diffusioncontrolled nucleation and growth along with the evolution and adherence of $\mathrm{H}_{2}$ bubbles on the $\mathrm{Cu}$ electrode under conditions of lower current densities, low voltages and low $\mathrm{Bi}^{3+}$ concentrations.

This facile and rational room temperature electrodeposition route can also be used for production of bimetallic or hybrid $\mathrm{Bi}$ based microstructures in combination with $\mathrm{Ag}$ and $\mathrm{Cu}$ (Figure 4).

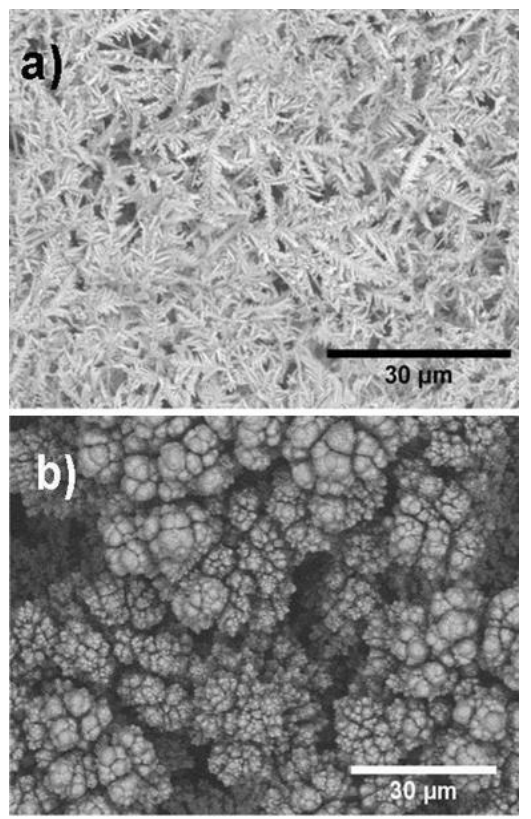

Figure 4. Representative E-SEM images of a) Bi-Ag dendritic, b) Bi-Cu broccoli bimetallic microstructures fabricated by the same electrodeposition methodology.

\section{Growth Mechanism}

In the present case the formation of subtle dendrites on a Cu wire during the initial stages can be interpreted by a mechanism originally proposed by Yang. ${ }^{[12 b]}$ It is governed by diffusioncontrolled nucleation and growth of $\mathrm{Bi}$ along with the evolution and adherence of $\mathrm{H}_{2}$ bubbles on the $\mathrm{Cu}$ electrode. The $\mathrm{H}_{2}$ bubbles direct the initial growth of the $\mathrm{Bi}$ dendrites. Electrochemical reactions with a $\mathrm{Cu}$ cathode in acidic solutions are accompanied by the evolution of $\mathrm{H}_{2}$. The hydrogen evolution reaction involves diffusion of $\mathrm{H}^{+}$to the metal (electrode) surface, reduction of $\mathrm{H}^{+}$to $\mathrm{H}$-atoms $\left(\mathrm{H}^{+}+\mathrm{e} \rightarrow \mathrm{H}\right)$, adsorption of $\mathrm{H}$-atoms on the metal surface, formation of molecular $\mathrm{H}_{2}\left(\mathrm{H}+\mathrm{H} \rightarrow \mathrm{H}_{2}\right)$, followed by the evolution of $\mathrm{H}_{2}$ bubbles from the surface. ${ }^{[18]}$ The activation polarization or overpotential for $\mathrm{H}^{+}$discharge on $\mathrm{Cu}$ $(0.44 \mathrm{~V})$ and $\mathrm{Bi}\left(0.40 \mathrm{~V}\right.$ at $10^{-2} \mathrm{mAmm}^{-2}$ current density) are in the same range in different acids. ${ }^{[19]} \mathrm{So}$, the $\mathrm{H}_{2}$ bubbles formed at the electrode surface are expected to cover most of the nucleation sites and suppress the further bismuth electrodeposition. However the $\mathrm{Bi}$ atoms have a poisoning effect. Chemically adsorbed $\mathrm{Bi}$ on $\mathrm{Pt}$ surfaces has been demonstrated to strongly reduce the rate of the hydrogen evolution reaction. ${ }^{[20]}$ Once a layer of $\mathrm{Bi}$ dendrite is formed, it prevents the formation of the $\mathrm{H}_{2}$ bubbles on the active sites of the bismuth deposit. There is a competition for electrons between $\mathrm{H}^{+}$and $\mathrm{Bi}^{3+}$. $\mathrm{Bi}$ atoms are known to block the $\mathrm{H}$-adsorption active sites and consume the electrons in its redox process. ${ }^{[20]}$

Moreover, in the present case strong cathodic overpotentials are used. At this increased overpotential, there is a high reduction rate of $\mathrm{Bi}^{3+}$ to $\mathrm{Bi}^{0}$. This leads to a very high nucleation density (increased number of crystals per unit area) and active sites. Thus, the consumption of $\mathrm{Bi}^{3+}$ may be more than their mass transport rate at the electrode-electrolyte interfacial region. Usually, this creates a depletion zone at the interface. In low concentration solutions (typically $1 \mathrm{mM}$ ) this mass-transport becomes a critical factor and limits the growth. However, the important factors in our deposition scheme involve the high concentration of the $\mathrm{Bi}^{3+}$ ions in solution and high current density. $\mathrm{A}$ very speedy growth of the electrically conducting $\mathrm{Bi}$ branches into the solution takes place. This rate is at about $50 \mu \mathrm{ms}^{-1}$. So here the mass transport of ions to the surface is not a limiting factor. Instead, the surface gets a continuous and rapid supply of reduced ions and the conducting branches very rapidly grow into the highly concentrated regions of the solution. Here the main driving process is reduction-controlled. A similar distinctive growth of $\mathrm{Zn}$ hexagons and their self-organisation into leaf-like structures has been demonstrated by López and Choi. ${ }^{[18 b]}$ This geometry optimization might also be explained by a fine balance between relatively low strain energies of the hexagonal form and a quite strong van der Waals interaction between the bismuth layers. ${ }^{[21]}$

\section{Characterization}

Quantitative measurements on the thickness of the Bi hexagons have been performed by atomic force microscopy (AFM) studies (Figure 5). The AFM image was obtained after transferring some of the smaller hexagons on a glass slide by exfoliation with water. Recently, Hernandez et al. ${ }^{[22]}$ have shown that graphite could be easily exfoliated in solvents like $\mathrm{N}$-methyl-pyrrolidone, whose surface energy is so well matched to that of graphene. Such exfoliation occurs because of the strong interaction between solvent and graphite. The energetic requirement for exfoliation and subsequent solvation is small. ${ }^{[2]}$ We suggest that similar but 
much weaker effects may occur between water and bismuth. Water has a surface energy of $0.072 \mathrm{Jm}^{-2}$ while for $\mathrm{Bi}$ the surface energy is found to be about $0.49 \mathrm{Jm}^{-2}$. ${ }^{[23]}$ Although there is a strong van der Waals interaction between the bismuth layers, ${ }^{[21]}$ but lower surface energy material (water) spontaneously wet the higher energy surface of $\mathrm{Bi}$ and is expected to reduce the interaction between the bismuth layers resulting is easy exfoliation. The AFM scan over an area of $80 \times 80 \mu \mathrm{m}^{2}$ (Figure $5 a$ ) and the line profile plot (Figure $5 b$ ) provide information on the vertical height of the hexagons and their topological roughness. The topological surface seems to be rather smooth and least undulated with a narrow height distribution while the mean height for hexagons is found to be about $900 \mathrm{~nm}$. A $1 \mu \mathrm{m}$ thick hexagon corresponds formally to about 2834 bilayers considering the distances between two $\mathrm{Bi}$ atoms in two neighbouring layers to be about $3.529 \AA^{[24]}$

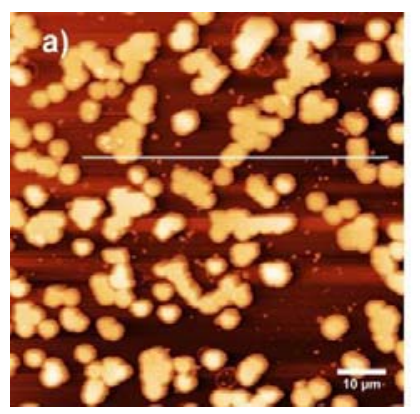

b)

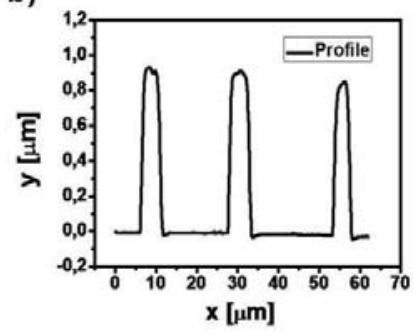

Figure 5. (Color online) a) AFM images of Bi particles transferred on a glass slide by exfoliation with water and $b$ ) the corresponding line-scan showing the height-profile.

The representative TEM image of the main dendritic trunk with protruding hexagons is shown in Figure $6 a$ and the HRTEM image of the attached hexagonal branch is shown in Figure $6 \mathrm{~b}$. The HRTEM image clearly reveals the presence of singular atomic planes which are aligned over the entire structure. The averaged interatomic spacing (inset of Figure $6 \mathrm{~b}$ ) is measured to be $3.2 \AA$ and indicates the high-quality single crystalline nature of the $\mathrm{Bi}$ hexagons. This calculated lattice spacing of the planes corresponds very well to the low energy (012) plane of rhombohedral $\mathrm{Bi}$ structure. The (012) growth direction in the trigonal $\mathrm{Bi}$ leading to a single-crystalline structure is apparently considered preferable for thermoelectric applications. ${ }^{[1 \mathrm{a}]}$ The selected area electron diffraction (SAED) pattern (insert of Figure 6b) also explicitly confirms the single-crystallinity of the $\mathrm{Bi}$ hexagons.
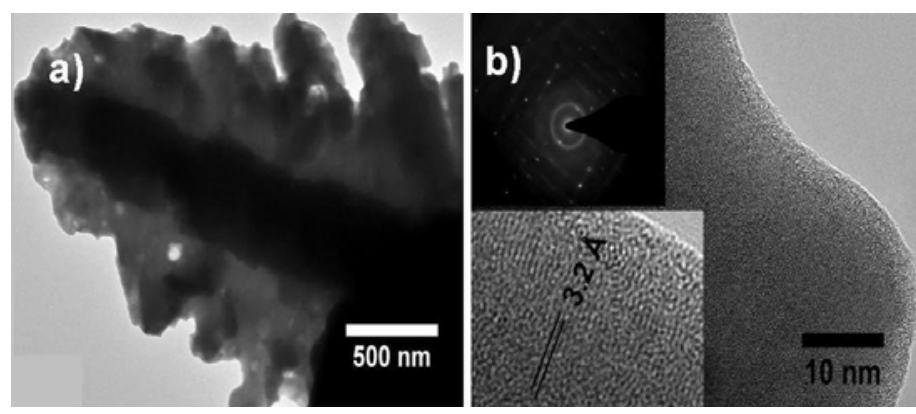

Figure 6. a) and b) representative TEM and HRTEM images. The inset of image $b$ shows the SAED of a Bi particle.
The elemental analysis of the electrodeposited $\mathrm{Bi}$ structures on a Cu-grid has been performed by energy-dispersive $\mathrm{X}$-ray (EDX) spectroscopy (Figure 7).

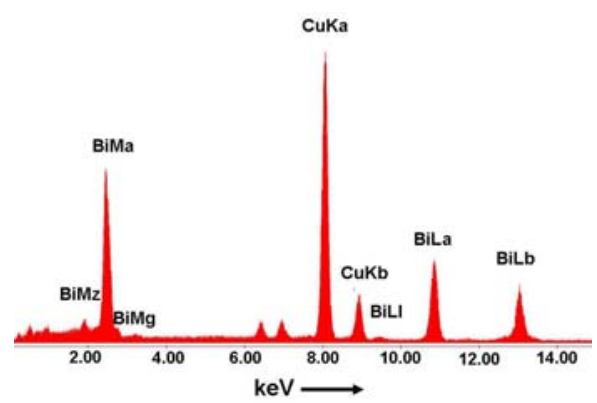

Figure 7. EDX spectrum of the electrodeposited $\mathrm{Bi}$. The $\mathrm{Cu}$ signals come from the TEM grid.

The XRD patterns of the water-washed $\mathrm{Bi}$ hexagons is shown in Figure 8, curve-a. All of the reflections can be readily indexed to a pure rhombohedral phase [space group: $R^{-} 3 m(166)$ ] of $\mathrm{Bi}$ compatible with the literature values of $a=4.546 \AA$ and $c=$ $11.862 \AA$ (JCPDS 85-1329). The Bi hexagonal motif crystals are found to be stable in air and in water under ambient conditions for several days. They are oxidized to yellow colored monoclinic $\alpha$ $\mathrm{Bi}_{2} \mathrm{O}_{3}$ (Figure 8, curve-b) (JCPDS card no.41-1449) only upon heat-treatment in air at $650{ }^{\circ} \mathrm{C}$ for $30 \mathrm{~min}$ with retention of hexagonal morphology.

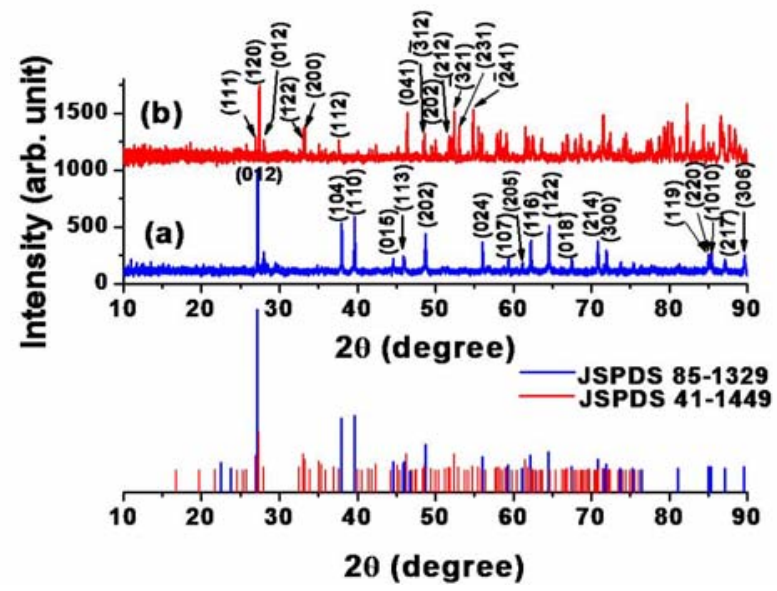

Figure 8. (Color online) XRD patterns of a) $\mathrm{Bi}$ hexagonal platelets b) $\mathrm{Bi}_{2} \mathrm{O}_{3}$ obtained after oxidation of $\mathrm{Bi}$ hexagons in air at $650{ }^{\circ} \mathrm{C}$ for $30 \mathrm{~min}$. The peak positions corresponding to that of rhombohedral $\mathrm{Bi}$ and monoclinic $\alpha-\mathrm{Bi}_{2} \mathrm{O}_{3}$ reported in literature (JCPDS: Joint Committee Powder Diffraction StandardsThe International Centre for Diffraction Data) are also given for comparison.

The Raman spectrum (Figure 9) of the Bi hexagons shows the bands at 69.9 and $96.2 \mathrm{~cm}^{-1}$. They arise due to the first-order scattering $E_{g}$ and $A_{1 g}$ modes of metallic $\mathrm{Bi}^{\left[{ }^{[25]}\right.}$ The inset shows the Raman spectrum of the thermally oxidized $\mathrm{Bi}$ at $650^{\circ} \mathrm{C}$ for $30 \mathrm{~min}$ All the bands correspond to the Raman signature of $\alpha-\mathrm{Bi}_{2} \mathrm{O}_{3}{ }^{[26]}$ It is thermodynamically the most stable form of all $\mathrm{Bi}_{2} \mathrm{O}_{3}$ polymorphs at room temperature having numerous photocatalytic applications. ${ }^{[25]}$ Controlled heat-treatment followed by slow cooling has negligible tendencies to generate mixed polymorphic phases. 


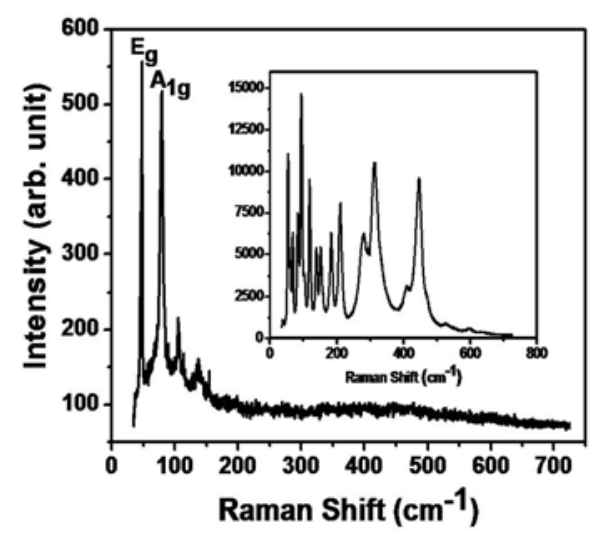

Figure 9. Raman spectrum of a single $\mathrm{Bi}$ hexagon. The inset shows the Raman spectrum of $\alpha-\mathrm{Bi}_{2} \mathrm{O}_{3}$ obtained after oxidation of $\mathrm{Bi}$ hexagons in air.

The energy dispersive X-ray (EDX) studies (Figure 7), powder X-ray diffraction (XRD) data (Figure 8), and Raman spectroscopic (Figure 9) measurements emphasize unambiguously the crystallinity, phase purity and metallic nature of the novel Bi hexagons.

\section{Thermal Stability of the Bi Hexagons}

Extended exposition to electrons in any electron microscope will force the bismuth hexagonal platelets to undergo deformation and cracking due to a slow melting process (Figure 10a-d). It was observed that smaller isolated hexagons can be formed by exfoliation with water (Figure 10e) which transform to small rounded octagons, polygons, disks or circular flowers and undergo agglomeration during electron beam irradiation (Figure 10f). A similar change of morphology upon exposure to an electron beam has also been reported for BiNi nano hexagons by Ould-Ely et al. ${ }^{[16 a]}$ It is interesting to note that the melting points for bulk $\mathrm{Bi}$ and $\mathrm{Bi}_{2} \mathrm{O}_{3}$ are 271 and $817{ }^{\circ} \mathrm{C}$, respectively. The melting of the $\mathrm{Bi}$ architectures under the electron beam, that is, at relatively low temperatures, indicates again the phase purity of the material. It also shows the need of future investigation of $\mathrm{Bi}$ crystals at cryogenic temperatures to ensure their long term thermal stability.

The $\mathrm{Bi}$ particles are also found to undergo melting upon exposure to very high intensity laser light (Figure 11). The selective spot-heating leads to in-situ oxidation as indicated by Raman spectroscopy.
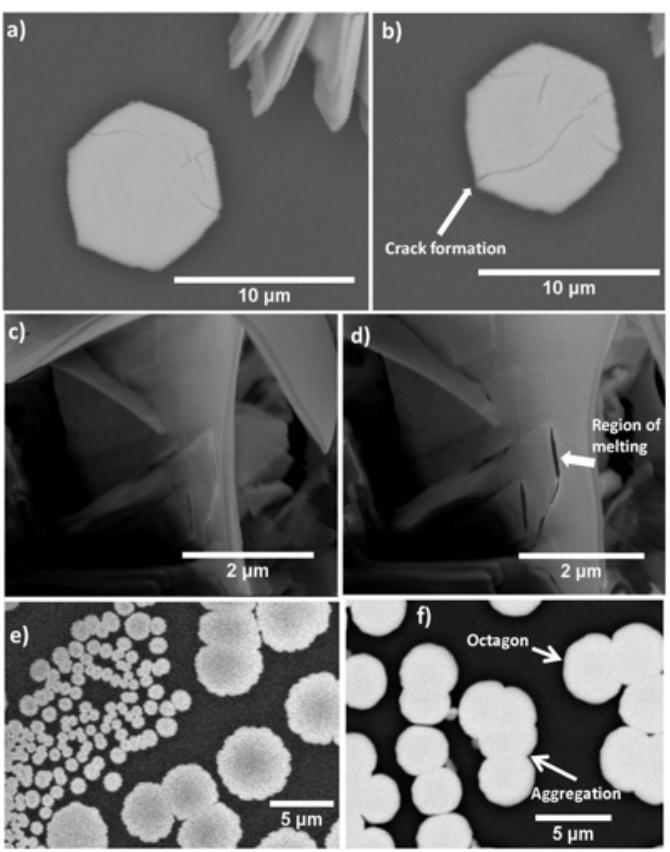

Figure 10. E-SEM images a) single Bi hexagon, b) crack formation in the $\mathrm{B}$ hexagon upon exposure to electron beam, c) Bi morphology, d) melting of $\mathrm{B}$ morphology upon irradiation with electron beam, e) Bi particles exfoliated with water on glass slide, and $\mathrm{f}$ ) aggregation and modification of structured $\mathrm{Bi}$ morphologies in presence of electron beam
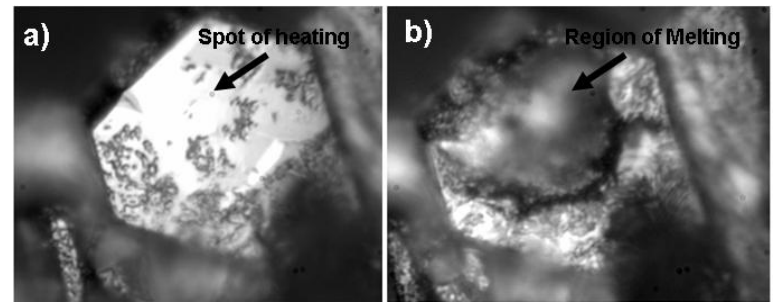

Figure 11. Optical microscopic image showing the melting of a single $\mathrm{B}$ hexagon by selective spot heating with a power of $250 \mathrm{~mW}$ of a $488 \mathrm{~nm} \mathrm{Ar}$ laser during Raman measurements within $90 \mathrm{~s}$.

\section{Chemical Stability Elemental Bi in Nitric Acid}

If the obtained electrodeposited products are not washed thoroughly with water, the incorporated nitric acid accelerates the production of a white basic polynuclear complex called bismuth oxide nitrate hydroxide hydrate $\mathrm{Bi}_{6}\left(\mathrm{NO}_{3}\right)_{4}(\mathrm{OH})_{2} \mathrm{O}_{6} \cdot 2 \mathrm{H}_{2} \mathrm{O}$ (JCPDS file no 8-06554). The formation is due to intramolecular polycondensation of hydrated $\mathrm{Bi}^{3+}$ ions. ${ }^{[27]}$ The process is fast and takes about a minute to complete.

The $\mathrm{Bi}$ structures are also found to re-dissolve in concentrated $\mathrm{HNO}_{3}$. The production of $\mathrm{Bi}$ micro-nano architectures in $\mathrm{HNO}_{3}$ electrolyte is actually a delicate competition between the two processes- deposition and re-dissolution; and is mainly determined by the strength of the acid. Most interestingly different morphologies have different dissolution rates depending on their exposed surface area, crystallinity and temperature. The barbed-wire dendrites consisting of small facetted crystals are found to have a higher dissolution rate in concentrated $\mathrm{HNO}_{3}$ than the isolated hexagons with a large surface area. So, when a mixture of morphologies is generated by electrodeposition, advantage of the selective re-dissolution phenomenon (in absence of current or voltage) can be undertaken to successfully and selectively generate a particular morphology intended for specific applications (Figure 12). 


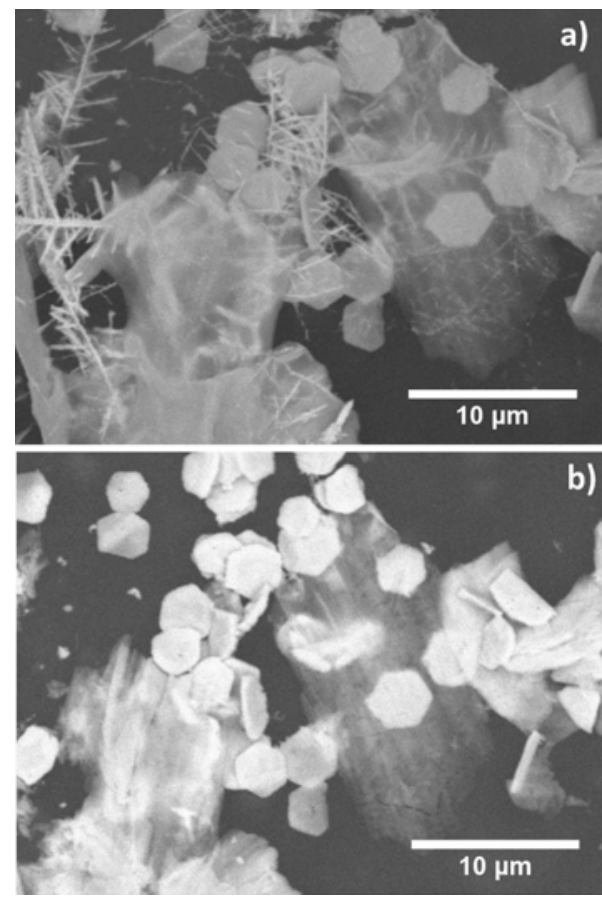

Figure 12. Representative E-SEM image of selective generation of $\mathrm{Bi}$ hexagons by discriminatory dissolution of dendritic $\mathrm{Bi}$ morphologies in $\mathrm{HNO}_{3}$. a) $\mathrm{Bi}$ hexagons and dendrites grown on Al surface, and b) dendrites dissolve while the hexagonal morphology is retained when dipped into concentrated $\mathrm{HNO}_{3}$ (1 M) for $20 \mathrm{~s}$. The hexagons are stable in $\mathrm{HNO}_{3}$ for several minutes. Images a and $\mathrm{b}$ show the sample before and after $\mathrm{HNO}_{3}$ treatment.

The formation and redissolution of the $\mathrm{Bi}$ structures with time in the same electrolyte (the life cycle of $\mathrm{Bi}$ hexagons in $\left.\mathrm{HNO}_{3}\right)$ is demonstrated in Figure 13. However, the rate of dissolution is exceedingly slow, by almost two orders of magnitude, as compared to the rate of electrodeposition (Figure 13). The dissolution rate is found to be slow and similar in $1 \mathrm{M}$ and $2 \mathrm{M} \mathrm{HNO}_{3}$ solution.

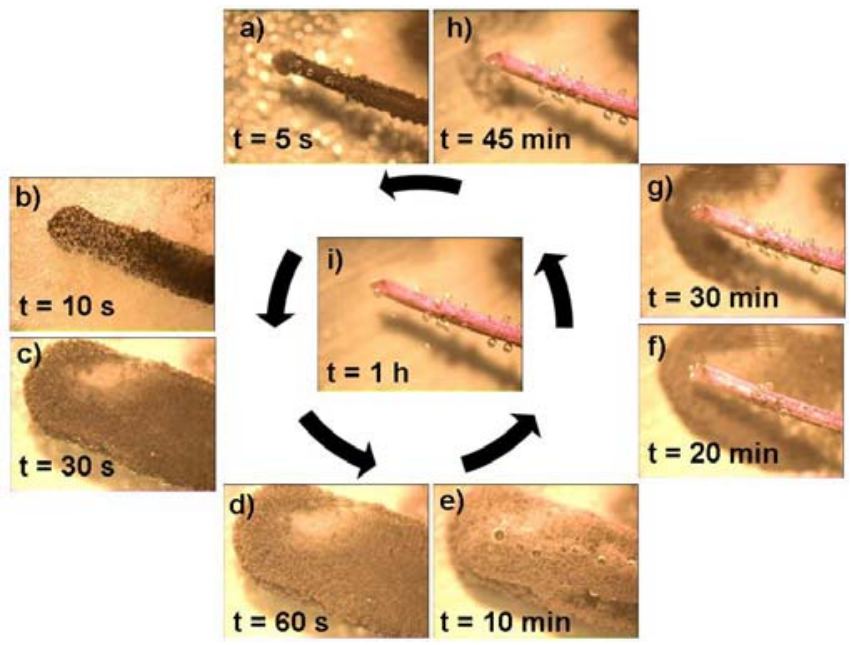

Figure 13. (Color online) a-d) Quick formation of $\mathrm{Bi}$ hexagons on a $\mathrm{Cu}$ wire $(0.4$ $\mathrm{mm}$ thick) at $\mathrm{V}=-10 \mathrm{~V}$ and $\mathrm{I}=300 \mathrm{~mA}$ in an electrolyte of $0.5 \mathrm{M} \mathrm{Bi}\left(\mathrm{NO}_{3}\right)_{3} \cdot 5 \mathrm{H}_{2} \mathrm{O}$ in $1 \mathrm{M} \mathrm{HNO}_{3}$. e-i) Very slow redissolution of the isolable $\mathrm{Bi}$ hexagons in the same electrolyte at $V=0$ and $I=0$. After 20 min (see image $\mathrm{f}$ ) the $\mathrm{Bi}$ deposits is detatched completely from the $\mathrm{Cu}$ wire and is subsequently dissolved. The dissolution is complete after $1 \mathrm{~h}$. Only the shadow of the $\mathrm{Cu}$ wire can be seen on the Petri dish (image i). A second cycle of rapid formation and slow dissolution of $\mathrm{Bi}$ hexagons could start on the same $\mathrm{Cu}$ wire.

\section{Thermoelectric Applications}

Crystalline thermoelectric materials exhibiting Seebeck voltages, which is the manifestation of a potential difference created by a temperature gradient across a material, have been in recent focus due to their waste heat-electricity and solar energy harvesting applications as alternatives to photovoltaics. ${ }^{[2]}$ The thermoelectric performance depends directly on the figure of merit $Z T=S^{2} \sigma T / K$, where $S$ is the Seebeck coefficient, $\sigma$ the electrical conductivity, $\mathrm{K}$ the thermal conductivity and $\mathrm{T}$ is the temperature. Pure bulk $\mathrm{Bi}$ is known to exhibit a negative Seebeck coefficient while $\mathrm{Sb}$ exhibits a positive value.$^{[4]}$ The $\mathrm{Bi}$ hexagons exhibit a greater drop in the negative Seebeck voltage upon application of the same temperature gradient (Figure 14). The preliminary measurement clearly demonstrates the better thermoelectric properties of assembled $\mathrm{Bi}$ hexagons than bulk $\mathrm{Bi}$. They are promising for fabrication of thermoelectric devices on the micro-scale. It is expected that the thermoelectric property of an isolated single-crystalline hexagon would be much better than the bulk. ${ }^{[1 b]}$ Two-dimensional exfoliated bismuth hexagons are expected to exhibit a high carrier mobility and a highly anisotropic Fermi surface.

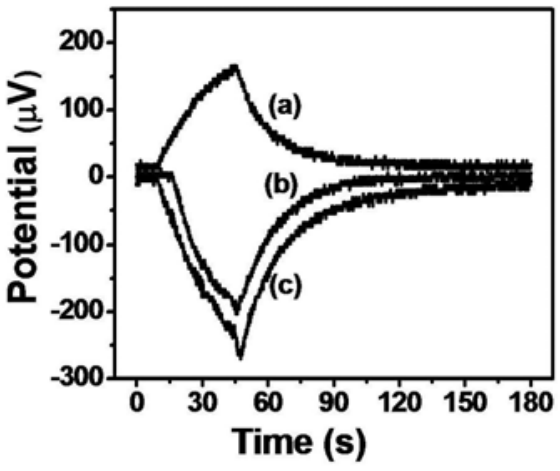

Figure 14. Potential change of a) bulk $\mathrm{Sb}, \mathrm{b}$ ) bulk $\mathrm{Bi}$ and c) $\mathrm{Bi}$ hexagons (pressed as a pellet) with time upon application of a constant heat source which resulted in a temperature difference of $\Delta T \approx 3 \mathrm{~K}$ after $45 \mathrm{~s}$. After $45 \mathrm{~s}$ the heat source was removed and the system was allowed to equilibrate.

\section{Catalytic Applications}

The $\mathrm{Bi}$ hexagons are also found to catalyze the reduction of 4nitrophenol (4-NP) to 4-aminophenol (4-AmP) by $\mathrm{NaBH}_{4}$ under ice-cold conditions. This reaction has been used frequently to check the catalytic activity of the various free or immobilized noble and transition metal particles in aqueous solution. ${ }^{[28]}$ However, to the best of our knowledge, it has never been used to study the catalytic efficiency of $\mathrm{Bi}$ particles. Under neutral or acidic conditions, 4-NP solution exhibits a strong absorption peak at $317 \mathrm{~nm}$. Upon the addition of $\mathrm{NaBH}_{4}$, the alkalinity of the solution increases resulting in 4-nitrophenolate ions as the predominant species and the color of the solution changes from light greenish-yellow to dark yellow. This results in a red shift of the absorption peak to about $400 \mathrm{~nm}$. With the addition of $\mathrm{Bi}$ hexagons as catalyst, the intensity of the absorption peak at 400 $\mathrm{nm}$ gradually decreases as the reduction reaction progresses. There is continuous fading of color leading to decoloration of the solution. The ratio of the absorbance $A_{t}$ and $A_{0}$ at times $t$ and 0 are measured from the relative intensity of the respective absorbances at $400 \mathrm{~nm}$ and $\ln \left(A_{t} / A_{0}\right)$ versus time is plotted for 
different catalyst concentrations (Figure 15). Approximately linear relationships are observed. The reaction rates, evaluated from the slope, are found to increase with increase in catalyst concentration. It is found to be $3.7 \times 10^{-5} \mathrm{~s}^{-1}\left( \pm 1 \times 10^{-6}\right), 1.9 \times 10^{-4}$ $\mathrm{s}^{-1}\left( \pm 5 \times 10^{-6}\right)$ and $3.1 \times 10^{-4} \mathrm{~s}^{-1}\left( \pm 4 \times 10^{-6}\right)$ for catalyst concentration of 100,500 and $1000 \mathrm{mgL}^{-1}$, respectively. In absence of catalyst no reaction was observed over several days. The formation of 4-AmP is marked by the evolution of a smal shoulder at $315 \mathrm{~nm}$. 4-AmP is an important industrial intermediate in the preparation of several analgesic and antipyretic drugs such as paracetamol, acetanilide and phenacetin. Therefore the direct catalytic reduction of 4-NP to 4-AmP by $\mathrm{NaBH}_{4}$ in presence of cheap, easily producible, surfactant-free Bi particles (washed and re-usable) could be a smart, efficient and sustainable methodology

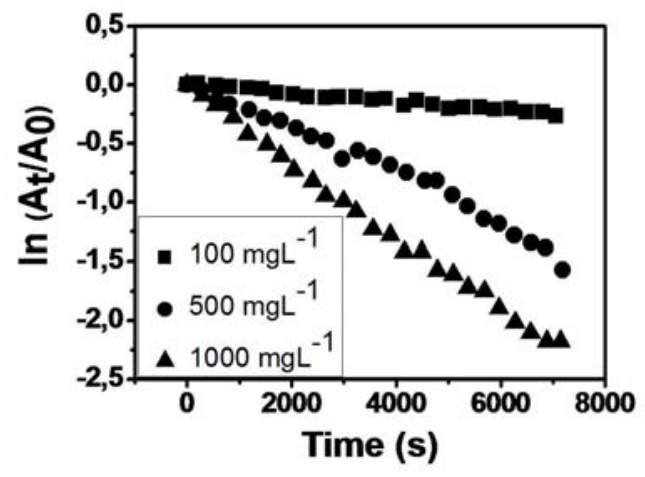

Figure 15. Plot of $\ln \left(A_{t} / A_{0}\right)$ versus time for different Bi hexagon concentrations

\section{Conclusion}

In summary, high purity $\mathrm{Bi}$ hexagonal single-crystals on a large scale has been successfully synthesized by single-step electrodeposition methodology within a short time interval (less than $1 \mathrm{~min}$ ) from nitric acid solutions in absence of any surfactant or complexing agents. This new electrodeposition protocol employing high current densities, high voltages and high concentrations at room temperature has several advantages. The main advantage of this method is that the Bi particles are easily isolable. These water-washed particles are found to be stable for several days under ambient conditions. These surfactant-free particles can be directly used for thermoelectric and catalytic applications. Indeed, this new generation material is found to be very promising for thermoelectric applications and catalysis. The material has been well characterized by SEM, AFM, TEM, EDX, $\mathrm{XRD}$ and Raman spectroscopy. The tendency of metallic $\mathrm{Bi}$ to form hexagonal platelets may be associated with its layered structure. The formation of hexagons under controlled electrolytic conditions could be attributed to the high reduction rate of $\mathrm{Bi}^{3+}$ ions and rapid growth of the $\mathrm{Bi}$ branches into a highly concentrated solution. Another novel finding is that the various morphologies of the same material (hexagons and dendrites) can undergo highly selective dissolution in nitric acid. These $\mathrm{Bi}$ hexagons have been oxidized to $\alpha-\mathrm{Bi}_{2} \mathrm{O}_{3}$ hexagons (by annealing at $650^{\circ} \mathrm{C}$ in air for $30 \mathrm{~min}$ ) which further have intriguing optoelectronic, photocatalytic and photovoltaic applications.

\section{Experimental Section}

Preparation of electrolytic solutions: Analytical grade purity bismuth nitrate pentahydrate (Aldrich) and nitric acid (JT Baker) were used. The $\mathrm{Bi}\left(\mathrm{NO}_{3}\right)_{3} \cdot 5 \mathrm{H}_{2} \mathrm{O}$ was first dissolved in concentrated $\mathrm{HNO}_{3}$ to prevent the formation of white precipitates of bismuth hydroxides and then diluted with deionized water.

The electrodeposition processes were carried out in an electrolyte of $0.5 \mathrm{M} \mathrm{Bi}\left(\mathrm{NO}_{3}\right)_{3} \cdot 5 \mathrm{H}_{2} \mathrm{O}$ and $1 \mathrm{M} \mathrm{HNO}_{3}$. This stock solution was kept at $298 \mathrm{~K}$ in ambient air. For the galvanostatic deposition, a power supply of Delta Electronics E 016-0.6 (Germany) was employed.

Instruments and characterization: SEM images of the samples were obtained using a TM-1000 tabletop environmental scanning electron microscope (Hitachi, Japan) while the FESEM images were obtained using a FEI Dual-Beam NOVA 600 field emission scanning electron microscope (NanoLab, The Netherlands). TEM images of the $\mathrm{Bi}$ particles grown directly on the $\mathrm{Cu}$ grids were taken with a CM200 FEG (Philips / FEI, The Netherlands) operating at $200 \mathrm{kV}$ acceleration voltage. The powder X-ray diffraction (XRD) measurements were carried out in the range $2 \theta=10-90^{\circ}$ using D8 Discover (Bruker AXS, Karlsruhe, Germany) with $\mathrm{Cu} \mathrm{K}_{\alpha 1}$ radiation $(\lambda=1.5374 \AA)$ with operating current of $40 \mathrm{~mA}$ and voltage of $40 \mathrm{kV}$, with scanning rate of 5 s/step. Micro Raman measurements were performed using a Princeton Instruments spectrometer (Roper Scientific, Germany) equipped with an $\mathrm{Ar}^{+}$laser operating at a wavelength of $488 \mathrm{~nm}$ obtained with a low power laser $(5 \mathrm{~mW})$. With increase in the intensity of the laser power beyond $100 \mathrm{~mW}$ and extended time of irradiation, the hexagonal crystals undergo deformation, start melting followed by in-situ oxidation due to selective spot heating. The AFM measurements were carried out with a Nanosurf® Mobile $S$ atomic force microscope (Nanoscience Instruments, USA). For thermoelectric property demonstration, the potential change within a period of $180 \mathrm{~s}$ was recorded with a VersaSTAT3 (Princeton Applied Research, AMETEK, Germany) using $\mathrm{Cu}$ electrodes sandwiching the same quantity of material and applying a constant heat source for 45 $\mathrm{s}$ which resulted in maximum temperature gradient of $\sim 3 \mathrm{~K}$. After $45 \mathrm{~s}$ the heat source was removed and the system was allowed to equilibrate. In a typical catalytic experiment, in $10 \mathrm{ml}\left(1.0 \times 10^{-4} \mathrm{M}\right)$ of aqueous solution of 4-nitrophenol, the $\mathrm{Bi}$ hexagons of different concentrations $\left(0,100,500\right.$ and $\left.1000 \mathrm{mgL}^{-1}\right)$ were added separately followed by addition of $5 \mathrm{ml}\left(6.0 \times 10^{-2} \mathrm{M}\right)$ of aqueous $\mathrm{NaBH}_{4}$ solution to the reaction mixture under ice-cold conditions and time-dependent absorption spectra were recorded with a HR2000 UV-vis spectrophotometer (Ocean Optics, Germany).

\section{Acknowledgements}

We are grateful to the financial support by the Deutsche Forschungsgemeinschaft (RA494/18-1, SPP 1415). TS thanks the Alexander von Humboldt Foundation, Germany for postdoctoral fellowship.

Keywords: Bismuth · Hexagons · Dendrites · Thermoelectric applications $\cdot$ Catalytic applications

[1] a) J. S. Son, K. Park, M.-K. Han, C. Kang, S. -G. Park, J. -H. Kim W. Kim, S. -J. Kim, T. Hyeon, Angew. Chem. Int. Ed. 2011, 50, 1363-1366; b) J. W. Roh, K. Hippalgaonkar, J. H. Ham, R. Chen, M. Z. Li, P. Ercius, A. Majumdar, W. Kim, W. Lee, ACS Nano 2011, 5, 3954-3960; c) A. Datta, G. S. Nolas, CrystEngComm, 2011, 13, 2753; d) D. Mott, N. T. Mai, N. T. B. Thuy, Y. Maeda, T. P. T. Linh, M. Koyano, S. Maenosono, Phys. Status Solidi A 2011, 208, 52-58.

[2] a) M. S. Dresselhaus, G. Chen, M. Y. Tang, R. Yang, H. Lee, D. Wang, Z. Ren, J.-P. Fleurial, P. Gogna, Adv. Mater. 2007, 19, 1043-1053; b) J. E. Moore, Nature 2010, 464, 194-198; c) J. Karni, Nature Mater. 2011, 10, 481-482; d) G. J. Snyder, E. S. Toberer, Nature Mater. 2008, 7, 105-114.

[3] a) D. Hsieh, D .Qian, L. Wray, Y. Xia, Y. S. Hor, R. J. Cava, M. Z. Hasan, Nature 2008, 452, 970-974; b) M. Z. Hasan, C. L. Kane, Rev. Mod. Phys. 2010, 82, 3045-3067; c) J. R. Sootsman, D. Y. 
Chung, M. G. Kanatzidis, Angew. Chem. Int. Ed. 2009, 48, 86168639; d) D. Kong, J. C. Randel, H. Peng, J. J. Cha, S. Meister, K. Lai, Y. Chen, Z. -X. Shen, H. C. Manoharan, Y. Cui, Nano Lett. 2010, 10, 329-333

[4] H. J. Goldsmid in Introduction to Thermoelectricity, Ch 9, Review of Thermoelectric Materials, (Eds.: R. Hull, J. Parisi, R. M. Osgood, Jr. H. Warlimont), Springer-Verlag Berlin, 2010.

[5] a) P.J. Kowalczyk, O. Mahapatra, D.N. McCarthy, W. Kozlowski, Z. Klusek, S.A. Brown, Surf. Sci. 2011, 605, 659-667; b) P. Hofmann, Prog. Surf. Sci. 2006, 81,191-245.

[6] a) S. Lee, J. Ham, K. Jeon, J. -S. Noh, W. Lee, Nanotech. 2010, 21 405701; b) S. Murakami, Phys. Rev. Lett. 2006, 97, 236805; c) M. Tian, J. Wang, Q. Zhang, N. Kumar, T. E. Mallouk, M. H. W. Chan, Nano Lett. 2009, 9, 3196-3202.

[7] a) F. Y. Yang, Kai Liu, Kimin Hong, D. H. Reich, P. C. Searson, C. L. Chien, Science 1999, 284, 1335-1337; b) G. Zhou, L. Li, G. H. Li, Appl. Phys. Lett. 2010, 97, 023112. c) M. Tian, J. Wang, Q. Zhang, N. Kumar, T. E. Mallouk, M. H. W. Chan, Nano Lett. 2009, 9, 31963202.

[8] a) A. Yella, E. Mugnaioli, M. Panthöfer, H. A. Therese, U. Kolb, W. Tremel, Angew. Chem. Int. Ed. 2009, 48, 6426 -6430; b) Y. Xiang, L. Cao, J. Arbiol, M. L. Brongersma, A. F. i Morral, Appl. Phys. Lett. 2009, 94, 163101; c) F. Wang, W. E. Buhro, Small 2010, 6, 573581.

[9] a) T.A. Hanna, Coord. Chem. Rev. 2004, 248, 429-440; b) M.T. Le J. Van Craenenbroeck, I. Van Driessche, S. Hoste, Appl. Catal. A 2003, 249, 355-364; c) W. Du, D. Su, Q. Wang, A. I. Frenkel, X. Teng, Cryst. Growth Design 2011, 11, 594-599.

[10] A. Luz, C. Feldmann, J. Mater. Chem., 2009, 19, 8107-8111.

[11] a) C. Kokkinos, A. Economou, I. Raptis, T. Speliotis, Electrochem Commun. 2011, 13, 391-395; b) H. Wang, Z. Yu, Z. Wang, H. Hao, Y. Chen, P. Wan, Electroanalysis 2011, 23, 1095-1099.

[12] a) Y. Ni, Y. Zhang, L. Zhang, J. Hong, CrystEngComm, 2011, 13, 794-799; b) M. Yang, J. Mater. Chem. 2011, 21, 3119-3124.

[13] a) M. Yerema, M. V. Kovalenko, G. Hesser, D. V. Talapin, W. Heiss, J. Am. Chem. Soc. 2010, 132, 15158-15159; b) F. Wang, R. Tang, H. Yu, P. C. Gibbons, W. E. Buhro, Chem. Mater. 2008, 20, 36563662; c) S. Sepulveda-Guzman, N. Elizondo-Villarreal, D. Ferrer, A Torres-Castro, X. Gao, J. P. Zhou, M. Jose-Yacaman, Nanotech. 2007, 18, 335604 .

[14] a) R. Boldt, M. Kaiser, D. Köhler, F. Krumeich, M. Ruck, Nano Lett. 2010, 10, 208-210; b) R. Fu, S. Xu, Y.-N. Lu, J. J. Zhu, Cryst. Growth Des. 2005, 5,1379-1385.

[15] a) J. Wang, X. Wang, Q. Peng, Y. Li, Inorg Chem 2004, 43, 75527556 ; b) W. Z. Wang, B. Poudel, Y. Ma, Z. F. Ren, J. Phys. Chem. B 2006, 110, 25702-25706; c) Y. Xu, Z. Ren, W. Ren, G. Cao, K Deng, Y. Zhong, Nanotech. 2008, 19,115602; d) S. Cao, C. Guo, Y Wang, J. Miao, Z. Zhang, Q. Liu, Solid State Commun. 2009, 149, $87-90$
[16] a) T. Ould-Ely, J. H. Thurston, A. Kumar, M. Respaud, W. Guo, C. Weidenthaler, K. H. Whitmire, Chem. Mater. 2005, 17, 4750-4754; b) W. Lu, Y. Ding, Y. Chen, Z. L. Wang, J. Fang, J. Am. Chem. Soc 2005, 127, 10112-10116; c) X. A. Fan, J. Y. Yang, Z. Xie, K. Li, W. Zhu, X. K. Duan, C. J. Xiao, Q. Q. Zhang, J. Phys. D: Appl. Phys. 2007, 40, 5975-5979; d) G. Pfaff, Special Effect Pigments, $2^{\text {nd }}$ revised edition, Vincentz Network, Hannover, 2008, pp 34-38.

[17] D. R. Lide, CRC Handbook of Chemistry and Physics, $76^{\text {th }}$ Edition, CRC Press, Boca Raton, 1995-1996, p. 8-22.

[18] a) M. P. Zach, K. H. Ng, R. M. Penner, Science 2000, 290, 21202123; b) F. Favier, E. Walter, M. P. Zach, T. Benter, R. M. Penner, Science 2001, 293, 2227-2231; c) C. M. López, K.-S. Choi, Langmuir 2006, 22, 10625-10629.

[19] R. W. Revie, H. H. Uhlig, Corrosion and Corrosion Control: An Introduction to Corrosion Science and Engineering, $4^{\text {th }}$ Edition Wiley Interscience, New Jersey, 2008, p. 62.

[20] R. Gómez, A. Fernández-Vega, J. M. Feliu, A. Aldaz, J. Phys. Chem 1993, 97, 4769-4776.

[21] B. Rasche, G. Seifert, A. Enyashin, J. Phys. Chem. C 2010, 114, 22092-22097.

[22] Y. Hernandez, et al. Nat. Nanotechnol. 2008, 3, 563-568.

[23] L. Vitos, A.V. Ruban, H.L. Skriver, J. Kollár, Surf. Sci. 1998, 411 186-202.

[24] Y. Li, J. Wang, Z. Deng, Y. Wu, X. Sun, D. Yu, P. Yang, J. Am Chem. Soc. 2001, 123, 9904-9905.

[25] a) X. Liu, H. Cao, J. Yin, Nano Res. 2011, 4, 470-482; b) S. Onari, M. Miura, K. Matsuishi, Appl. Surf. Sci. 2002, 197-198, 615-618.

[26] G. Lin, D. Tan, F. Luo, D. Chen, Q. Zhao, J. Qiu, Z. Xu, J. Alloys Compd. 2010, 507, L43-L46.

[27] N. Henry, O. Mentré, F. Abraham, E.J. MacLean, P. Roussel, J. Solid State Chem. 2006, 179, 3087-3094.

[28] N. Pradhan, A. Pal, T. Pal, Colloid Surf. A 2002, 196, 247-257; S. Saha, A.Pal, S. Kundu, S. Basu, T. Pal, Langmuir 2010, 26, 2885 2893; K. -L. Wu, X. -W. Wei, X. -M. Zhou, D. -H. Wu, X. -W. Liu, Y. Ye, Q. Wang, J. Phys. Chem. C 2011, 115, 16268-16274; S. Harish, J. Mathiyarasu, K. L. N. Phani, V. Yegnaraman, Catal. Lett. 2009, 128, 197-202.

Received: ((will be filled in by the editorial staff))

Published online: ((will be filled in by the editorial staff) 


\section{Entry for the Table of Contents}

Layout 1:

\section{ARTICLES}

The highly selective mass synthesis of stable, isolable, surfactant-free, single-crystalline $\mathrm{Bi}$ hexagons has been demonstrated by forceful electrodeposition on a $\mathrm{Cu}$ wire at room temperature by applying very high current densities, high voltages and high electrolyte concentrations quite contrary to the usual electrodeposition methodologies. The material is found to be promising for thermoelectric and catalytic applications.

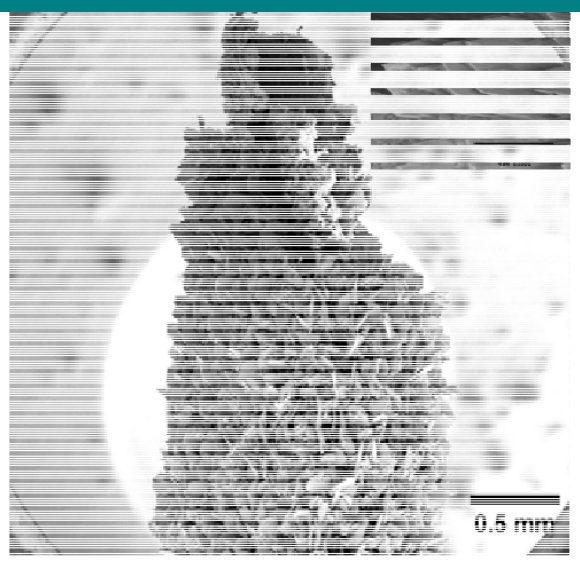

Tirtha Som, Anne Simo, Robert Fenger, Gerald V. Troppenz, Roman Bansen, Norbert Pfänder, Franziska Emmerling, Jörg Rappich, Torsten Boeck, and Klaus Rademann *

Page No. - Page No.

Bismuth Hexagons: Facile Mass Synthesis, Stability and Applications 